\title{
Oxidative stress and glucose metabolism-is there a need to revisit effects of insulin treatment?
}

\author{
P. M. Humpert
}

Received: 13 November 2009 / Accepted: 11 December 2009 /Published online: 6 January 2010

(C) Springer-Verlag 2009

Keywords Diabetes · Glycaemic variability · Insulin .

Oxidative stress · Vascular complications

$\begin{array}{ll}\text { Abbreviations } & \\ \text { 8-Iso-PGF2 } \alpha & \text { 8-Isoprostaglandin } \mathrm{F}_{2 \alpha} \\ \text { MAGE } & \text { Mean amplitude of glycaemic excursions } \\ \text { OHA } & \text { Oral hypoglycaemic agent }\end{array}$

Oxidative stress induced by hyperglycaemia and subsequent cellular damage is thought to be one of the major pathophysiological factors causing late complications in diabetes [1, 2]. Hyperglycaemia-dependent superoxide overproduction by the mitochondrial electron transport chain plays a key role in the cellular activation leading to endothelial damage. The generation of reactive oxygen species also involves an increased polyol and hexosamine pathway flux as well as activation of protein kinase $\mathrm{C}$ and excess formation of advanced glycation end-products [1]. These pathways lead to pro-inflammatory cell activation and subsequent endothelial dysfunction in diabetes $[1,3]$.

Even in healthy individuals, glucose challenges have been shown to increase formation of cellular oxidative stress [4] and trigger proinflammatory cellular activation [5]. In addition to hyperglycaemia itself, Monnier and colleagues previously defined glucose fluctuations as determinants of the amount of oxidative stress generated

\section{P. M. Humpert $(\triangle)$}

Department of Medicine I and Clinical Chemistry,

University of Heidelberg,

Im Neuenheimer Feld 410,

69120 Heidelberg, Germany

e-mail: Per.Humpert@med.uni-heidelberg.de in type 2 diabetes patients [6]; they showed that the mean amplitude of glycaemic excursions (MAGE) from continuous glucose monitoring data correlated positively and independently with urinary 8-iso prostaglandin $\mathrm{F}_{2 \alpha}$ (8-isoPGF2 $\alpha$ ) excretion as an indicator of oxidative stress [6] in patients with poorly controlled diabetes on oral hypoglycaemic agents (OHA). In this issue of Diabetologia, Monnier et al. report an influence of insulin therapy on urinary 8 -iso-PGF $2 \alpha$ excretion in a cross-sectional setting and provide data from small groups of type 2 diabetes patients started on either insulin therapy or receiving additional OHA [7]. The results from this study are particularly interesting, as they imply an independent effect of insulin therapy on oxidative stress and could help explain some recent observations on glucose variability and the development of late complications in clinical trials [8-10].

In the cross-sectional study, type 2 diabetes patients on OHA were shown to have a $\sim 60 \%$ increased urinary 8 -isoPGF $2 \alpha$ excretion compared with type 1 or type 2 diabetes patients treated with insulin. 8-Iso-PGF2 $\alpha$ excretion in the latter two groups did not differ significantly compared with non-diabetic controls. As reported in the previous publication [6], 8-iso-PGF $2 \alpha$ excretion was independently associated with MAGE and $24 \mathrm{~h}$ mean glucose levels in type 2 diabetes patients treated with OHA only. In addition, in the current study, 8-iso-PGF $2 \alpha$ excretion was independently associated with long-term glucose control reflected by $\mathrm{HbA}_{1 \mathrm{c}}$ [7]. However, these associations were not found in the insulin-treated type 1 and type 2 diabetes patients, further suggesting that insulin treatment itself inhibits oxidative stress in type 1 and type 2 diabetes, reducing it to levels observed in non-diabetic individuals [7]. Additional evidence for an antioxidant effect of insulin was 
added by longitudinal observations in type 2 diabetes patients. Initiation of insulin therapy significantly decreased 8 -iso-PGF $2 \alpha$ excretion, while no significant change in $\mathrm{HbA}_{1 \mathrm{c}}$, MAGE and mean $24 \mathrm{~h}$ glucose levels was seen. In contrast, patients given add-on OHA did not show any differences in 8-iso-PGF $2 \alpha$ excretion, despite a significant decrease of glucose variability and better long-term glucose control [7].

The reported data could be of great importance, since previous clinical endpoint studies have focused on the indirect metabolic effects of insulin therapy geared primarily to achieving pre-defined $\mathrm{HbA}_{1 \mathrm{c}}$ levels and reducing postprandial glucose excursions [9, 11]. The possible direct effects of insulin on oxidative stress and vascular function have remained out of the focus. There is, however, some clinical and experimental evidence supporting this possibility. In an experimental setting it has been shown that intracellular oxidative stress as reflected by the glutathione: glutathione disulfide ratio is diminished by insulin in type 2 diabetes patients undergoing a euglycaemic-hyperinsulinaemic clamp after only $60 \mathrm{~min}$ [12]. In vitro, in human aortic endothelial cells, insulin suppressed intercellular adhesion molecule-1 and monocyte chemoattractant protein-1 production, as well as NF-kB binding, which are known to be central mediators of inflammation and diabetic complications [3, 13, 14]; inhibition of NF-KB and of oxidative stress have also been shown to be downregulated by infusion of insulin in obese patients [15]. In patients with acute myocardial infarction, insulin infusion was shown to elicit antiinflammatory and pro-fibrinolytic effects [16]. Consequently, insulin improved arterial vasodilation, elasticity and limb perfusion by means of nitric oxide-dependent and -independent mechanisms in healthy controls as well as in type 2 diabetes patients [17-20]. In addition to these effects on inflammation and vascular function, insulin potentially influences vascular regeneration by stimulating and mobilising endothelial progenitor cells in vitro and in vivo [21-23]. Yet, insulin effects on cellular homeostasis could also depend on insulin concentrations, since supraphysiological doses of insulin have been shown to induce generation of reactive oxygen species in vitro [24]. Taken together, these data and the current results of Monnier and colleagues [7] imply that insulin itself could have protective effects on the vasculature. However, so far these glucoseindependent effects of insulin have not been studied in patients. Clinical trials comparing early insulin therapy and escalation of OHA in type 2 diabetes would be needed to address this question.

Glucose variability and postprandial glucose excursions are thought to influence the development of micro- and macrovascular late complications. Previous data and the current study by Monnier and colleagues show that MAGE is significantly associated with 8 -iso-PGF $2 \alpha$ excretion in patients with type 2 diabetes [6, 7]. It seems plausible that in these patients glucose variability increases oxidative stress, thereby influencing progression of the disease. Targeting of postprandial glucose values in the STOPNIDDM trial did indeed reduce the development of diabetes, hypertension and cardiovascular events [25, 26]. Yet, glucose variability was not identified as a risk factor for microvascular disease or neuropathy in the DCCT study of type 1 diabetes patients treated with insulin $[8,10]$, while the HEART-2D trial failed to show that prandial insulin therapy is superior to a basal regimen in the prevention of secondary cardiovascular events [9]. These considerations and the observation that insulin therapy improves oxidative stress to levels observed in healthy non-diabetic patients [7] prompts speculation that glucose variability does not contribute significantly to the progression of vascular complications in diabetes patients on insulin treatment in whom insulin itself could elicit beneficial effects on the vessel. Most interestingly in this context, recent data from the Pittsburgh Epidemiology of Diabetes Complications study in type 1 diabetes showed a glucose-independent association between lower insulin dose at baseline and the development of non-fatal myocardial infarction [27]. However, these considerations remain speculative and should be followed up in controlled clinical trials.

The observational design of the study by Monnier and colleagues [7] means that data should be interpreted with caution. Thus the cross-sectional patient groups are unbalanced and do not match well, especially with regard to age, vascular risk factors and prevalent late complications [7]. In addition, urinary 8 -iso-PGF $2 \alpha$ excretion has not so far been established as a marker for prediction of complications in diabetes and vascular disease, while the concept that urinary 8-iso-PGF $2 \alpha$ excretion resembles oxidative burden is widely accepted [28]. Future studies will have to show whether other direct and indirect markers of oxidative stress and inflammation support the data obtained in this study. The exact mechanisms of insulin's vascular effects and its anti-oxidative capacity need to be clarified, since the current study by Monnier and colleagues [7] cannot discriminate direct and indirect effects of insulin.

It is time to revisit insulin effects. Especially in type 2 diabetes, different treatment strategies need to be studied with a focus on macrovascular endpoints and myocardial function. Associations between hyperinsulinaemia and vascular events seem to be largely explained by confounding factors [29], while recent data from type 1 diabetes patients suggest that future interventional and epidemiological studies should adjust for the possibility that insulin dosage along with markers of glucose control could influence outcomes [27]. The question of whether insulin therapy should be initiated early in the course of type 2 diabetes and which regimen should be applied cannot be 
answered at this point. However, insulin therapy might be superior to a polypharmacological approach as long as effects of novel substances on vascular late complications remain unclear.

Duality of interest The author declares that there is no duality of interest associated with this manuscript.

\section{References}

1. Brownlee M (2005) The pathobiology of diabetic complications: a unifying mechanism. Diabetes 54:1615-1625

2. Evans JL, Goldfine ID, Maddux BA, Grodsky GM (2002) Oxidative stress and stress-activated signaling pathways: a unifying hypothesis of type 2 diabetes. Endocr Rev 23:599-622

3. Bierhaus A, Humpert PM, Morcos M et al (2005) Understanding RAGE, the receptor for advanced glycation end products. J Mol Med 83:876-886

4. Mohanty P, Hamouda W, Garg R, Aljada A, Ghanim H, Dandona $P$ (2000) Glucose challenge stimulates reactive oxygen species (ROS) generation by leucocytes. J Clin Endocrinol Metab 85:2970-2973

5. Schiekofer S, Andrassy M, Chen J et al (2003) Acute hyperglycemia causes intracellular formation of CML and activation of ras, p42/44 MAPK, and nuclear factor kappaB in PBMCs. Diabetes 52:621-633

6. Monnier L, Mas E, Ginet C et al (2006) Activation of oxidative stress by acute glucose fluctuations compared with sustained chronic hyperglycemia in patients with type 2 diabetes. Jama 295:1681-1687

7. Monnier L, Colette C, Mas E et al (2009) Regulation of oxidative stress by glycaemic control: evidence for an independent inhibitory effect of insulin therapy. Diabetologia. doi:10.1007/ s00125-009-1574-6

8. Kilpatrick ES, Rigby AS, Atkin SL (2009) Effect of glucose variability on the long-term risk of microvascular complications in type 1 diabetes. Diabetes Care 32:1901-1903

9. Raz I, Wilson PW, Strojek K et al (2009) Effects of prandial vs fasting glycemia on cardiovascular outcomes in type 2 diabetes: the HEART2D trial. Diabetes Care 32:381-386

10. Siegelaar SE, Kilpatrick ES, Rigby AS, Atkin SL, Hoekstra JB, Devries JH (2009) Glucose variability does not contribute to the development of peripheral and autonomic neuropathy in type 1 diabetes: data from the DCCT. Diabetologia 52:2229-2232

11. Del Prato S (2009) Megatrials in type 2 diabetes. From excitement to frustration? Diabetologia 52:1219-1226

12. Bravi MC, Armiento A, Laurenti O et al (2006) Insulin decreases intracellular oxidative stress in patients with type 2 diabetes mellitus. Metabolism 55:691-695

13. Bierhaus A, Nawroth PP (2009) Multiple levels of regulation determine the role of the receptor for AGE (RAGE) as common soil in inflammation, immune responses and diabetes mellitus and its complications. Diabetologia 52:2251-2263
14. Aljada A, Ghanim H, Saadeh R, Dandona P (2001) Insulin inhibits NFkappaB and MCP-1 expression in human aortic endothelial cells. J Clin Endocrinol Metab 86:450-453

15. Dandona P, Aljada A, Mohanty $P$ et al (2001) Insulin inhibits intranuclear nuclear factor kappaB and stimulates IkappaB in mononuclear cells in obese subjects: evidence for an antiinflammatory effect? J Clin Endocrinol Metab 86:3257-3265

16. Dandona P, Chaudhuri A, Ghanim H, Mohanty P (2009) Insulin as an anti-inflammatory and antiatherogenic modulator. J Am Coll Cardiol 53:S14-S20

17. Steinberg HO, Brechtel G, Johnson A, Fineberg N, Baron AD (1994) Insulin-mediated skeletal muscle vasodilation is nitric oxide dependent. A novel action of insulin to increase nitric oxide release. J Clin Invest 94:1172-1179

18. Tamminen $\mathrm{M}$, Westerbacka J, Vehkavaara S, Yki-Jarvinen $\mathrm{H}$ (2002) Insulin-induced decreases in aortic wave reflection and central systolic pressure are impaired in type 2 diabetes. Diabetes Care 25:2314-2319

19. Westerbacka J, Wilkinson I, Cockcroft J, Utriainen T, Vehkavaara S, Yki-Jarvinen H (1999) Diminished wave reflection in the aorta. A novel physiological action of insulin on large blood vessels. Hypertension 33:1118-1122

20. Vehkavaara S, Makimattila S, Schlenzka A, Vakkilainen J, Westerbacka J, Yki-Jarvinen H (2000) Insulin therapy improves endothelial function in type 2 diabetes. Arterioscler Thromb Vasc Biol 20:545-550

21. Humpert PM, Djuric Z, Zeuge U et al (2008) Insulin stimulates the clonogenic potential of angiogenic endothelial progenitor cells by IGF-1 receptor-dependent signaling. Mol Med 14:301-308

22. Humpert PM, Neuwirth R, Battista MJ et al (2005) SDF-1 genotype influences insulin-dependent mobilization of adult progenitor cells in type 2 diabetes. Diabetes Care 28:934-936

23. Fadini GP, Baesso I, Agostini C et al (2008) Maternal insulin therapy increases fetal endothelial progenitor cells during diabetic pregnancy. Diabetes Care 31:808-810

24. Ceolotto G, Bevilacqua M, Papparella I et al (2004) Insulin generates free radicals by an $\mathrm{NAD}(\mathrm{P}) \mathrm{H}$, phosphatidylinositol 3'kinase-dependent mechanism in human skin fibroblasts ex vivo. Diabetes 53:1344-1351

25. Chiasson JL, Josse RG, Gomis R, Hanefeld M, Karasik A, Laakso $M$ (2002) Acarbose for prevention of type 2 diabetes mellitus: the STOP-NIDDM randomised trial. Lancet 359:2072-2077

26. Chiasson JL, Josse RG, Gomis R, Hanefeld M, Karasik A, Laakso M (2003) Acarbose treatment and the risk of cardiovascular disease and hypertension in patients with impaired glucose tolerance: the STOP-NIDDM trial. Jama 290:486-494

27. Conway B, Costacou T, Orchard T (2009) Is glycaemia or insulin dose the stronger risk factor for coronary artery disease in type 1 diabetes? Diab Vasc Dis Res 6:223-230

28. Patrignani P, Tacconelli S (2005) Isoprostanes and other markers of peroxidation in atherosclerosis. Biomarkers 10(Suppl 1):S24S29

29. Lakka HM, Lakka TA, Tuomilehto J, Sivenius J, Salonen JT (2000) Hyperinsulinemia and the risk of cardiovascular death and acute coronary and cerebrovascular events in men: the Kuopio Ischaemic Heart Disease Risk Factor Study. Arch Intern Med 160:1160-1168 